\title{
Tree Automata for Schema-level Filtering of XML Associations
}

\author{
Vaibhav Gowadia and Csilla Farkas \\ Information Security Laboratory \\ Department of Computer Science and Engineering \\ University of South Carolina, Columbia, SC 29208 \\ \{gowadia, farkas \}@cse.sc.edu
}

\begin{abstract}
In this paper we present query filtering techniques based on bottomup tree automata for XML access control. In our authorization model (RXACL), RDF statements are used to represent security objects and to express the security policy. We present the concepts of a simple security object and an association security object. Our model allows us to express and enforce access control on XML trees and their associations. We propose a query-filtering technique that evaluates XML queries to detect disclosure of association-level security objects. We use tree automata to model-security objects. Intuitively a query $Q$ discloses a security object $o$ if and only if the (tree) automata corresponding to $o$ accepts $Q$. We show that our schema-level method detects all possible disclosures, i.e., it is complete.

Keywords: Access control, association object, flexible security granularity, XML security, tree automata.

ACM Classification: H.2.7 (Database Management - Database Administration - security, integrity and protection), D.4.6 (Operating Systems Security and Protection - Access controls)
\end{abstract}




\section{Introduction}

Several XML access control models have been developed recently (Bertino et al., 2001, 1999, 2000; Damiani et al., 2000; Dridi and Neumann, 1998; Gabillon and Bruno, 2002; Kudo and Hada, 2000; Murata et al., 2003; Luo et al., 2004). They are based on traditional access control lists and provide extensions to XML syntax. Existing models allow node-level security granularity by assigning access restrictions to the nodes and links of XML documents. However, none of these models provide access control for data associations. Intuitively, an association security object is an XML subtree that is not allowed to be accessed by a user, while all of its proper subtrees are permitted separately. Incorporating association in an access control model increases data availability while preserving confidentiality.

To illustrate the need of access control for data associations, we present an example in the medical domain. Assume that XML format is used for storing patient records. The DTD for patients' health records is shown in Fig. 1. Alice, who is an intern at the hospital, needs limited access to the database. Her duties involve two main tasks: 1) Alice contacts patients to collect feedback about their treatments; thus, Alice is allowed to read $<$ name $>$ and $<$ PhoneNumber $>$ elements, 2) Alice prepares statistical reports based on Age, Race and Diagnosis of the patients. These tasks require that Alice is allowed to access both contact and diagnosis information for all patients. However, Alice is not authorized to access data about the name and diagnosis of the patients. Both, the functionality requirements of Alice's work and the security restrictions cannot be satisfied using traditional access control list based methods.

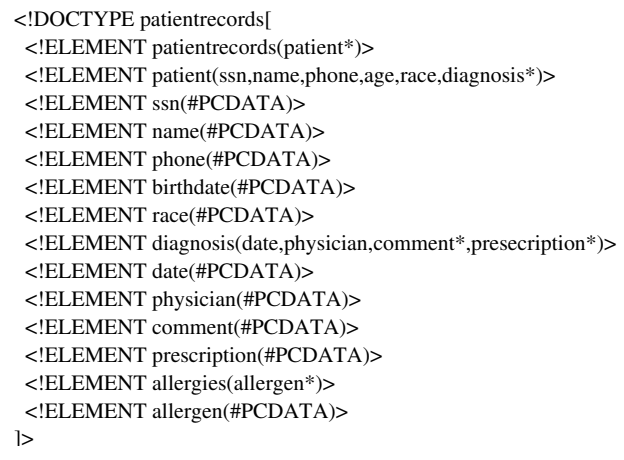

(a)

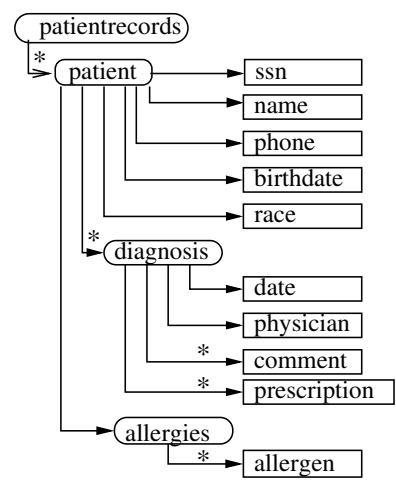

(b)

Figure 1: (a) An example of a DTD (b) Tree representation of DTD

The RXACL architecture, introduced in Gowadia and Farkas (2003), provides flexible access control granularity by allowing security classification of XML nodes and subtrees (simple security objects), and associations among 
nodes (association security objects). In Gowadia and Farkas (2003) we proposed a technique to enforce association-based access control at data-level (i.e., check for security violation after query processing) and it is outside the scope of this paper. In this paper we extend RXACL architecture by presenting techniques for performing a security check before the query is processed. Our work is similar to those proposed by Murata et al. (2003) and Luo et al. (2004). However, their methods support node-level security objects only. The automata model, used by them, is not sufficient to model association-level security objects. In this paper, we use bottom-up tree automata to represent security objects.

We propose query pre-processing techniques to recognize disclosure of association level security objects by XML queries. This analysis is data-independent. Results of query pre-processing can be (1) association objects are disclosed, (2) association objects are not disclosed, or (3) association objects may be disclosed. Options 1 and 2 indicate that the query should be rejected or accepted, respectively. For option 3, data-level analysis is required to evaluate whether a security violation occurs or not.

We present a two-layered association filtering method. First we detect disclosure of association in a given query-pattern, i.e., in information encoded in the XML query itself. Second, we extend query-pattern with document schema to represent all schema information that the query answer would reveal to a user. XML query-patterns are labeled-trees where node labels may be variables, constants, or the special symbol '//'(self-or-descendant axis (Clark and DeRose, 1999)). We model association security objects with pattern automata (Definition 3.8). A pattern automata takes (extended) query-patterns as input and accepts them if and only if the input discloses the security object represented by the pattern automata. The main technical contributions of this paper are the development of pattern automata for security objects and the notion of extended query-pattern. We present algorithms to construct query-pattern, pattern automata, and to detect disclosure of security objects.

The organization of the paper is as follows: next section presents an overview of RXACL architecture and query filtering mechanism. Section 3 introduces formal definitions of basic constructions used in this paper. Section 4 presents algorithms for constructing query-patterns, association pattern automata and to detect association disclosures. Section 5 introduces the notion of extended query-pattern and presents a schema-level security analysis of query. Our conclusions are presented in section 6 along with recommendations for future work.

\section{RDF-based XML Access Control Architecture}

Figure 2 shows the RXACL architecture. The architecture contains four main components: 1. Query filter 2. Query engine, 3. Data level access control, and 4. User history. The query filtering component performs schema-level analysis to determine whether an answer to the input query : (1) violates access control policy (violating), (2) does not violate the access control policy (safe), or (3) requires a data-level security check to detect possible violations (unsafe). The 


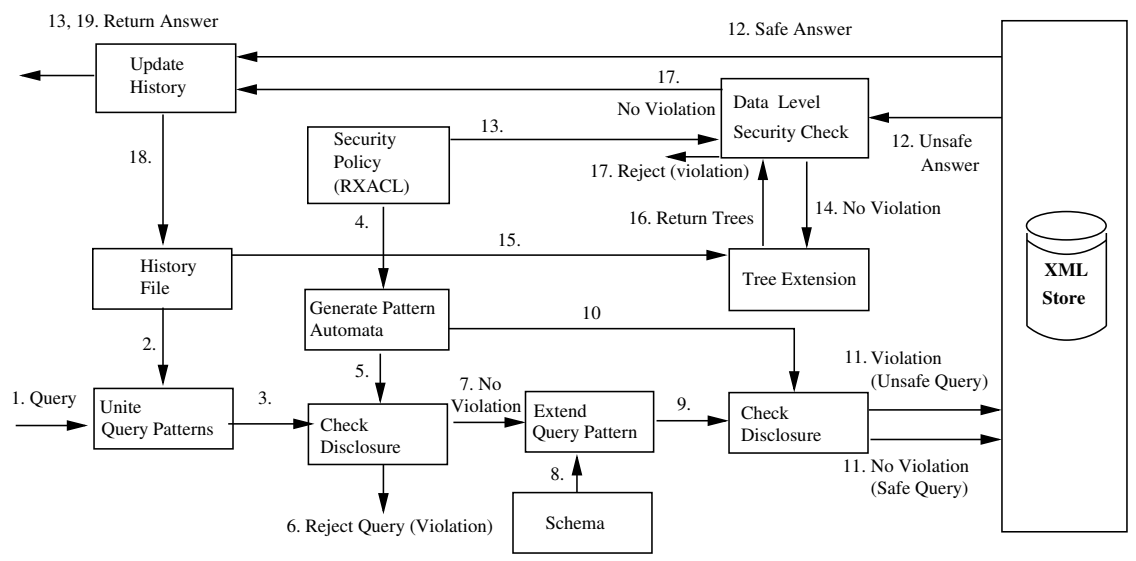

Figure 2: RXACL architecture for enforcing XML access control

XML query engine is responsible for generating responses to user's requests. RXACL uses an existing XML query engine, the development of such an engine is outside the scope of this paper. The data-level access control component analyzes the query-answer based on the security policy and data previously released to the user (Gowadia and Farkas, 2003). The history component keeps track of answered query-patterns and data released to each user.

When a data request is submitted to a RXACL system, query filtering component first checks for disclosure of disallowed association-level security objects in the query (without utilizing the XML schema information). If a disallowed association-level security object is disclosed, the query is immediately rejected. Otherwise, the query-pattern is extended with schema information and querypatterns of previously answered queries to the user. Extended query-patterns are now checked for disallowed objects. If no disallowed association-level security object is disclosed in extended query-patterns the query answer is labeled safe. Otherwise, it is labeled unsafe. The query is submitted to XML query engine for further processing. The result of unsafe query's evaluation are further evaluated for possible data-level violations as described in Gowadia and Farkas (2003). Answer to safe queries is returned to user without further security analysis. For all queries that are answered, user history is updated with query-pattern and released data. The assurance of our query level filtering is based on the completeness property of the filtering.

\section{Definitions}

This section describes definitions necessary to model XML queries, association objects, and XML schema.

Definition 3.1 (Labeled-tree) 
A labeled-tree, or a tree, is defined recursively as follows:

1. The empty set \{\} is a tree, called the empty tree.

2. A single node $\{n\}$ is a tree.

3. If $t_{1}, t_{2}, \ldots, t_{k}$ are trees, then $\left\{n \rightarrow\left\{t_{1}, t_{2}, \ldots, t_{k}\right\}\right\}$ is a tree. In this case we say that $\left\{n \rightarrow\left\{t_{1}, t_{2}, \ldots, t_{k}\right\}\right\}$ represents the tree whose root $n$ has outgoing edges to subtrees $t_{1}, t_{2}, \ldots, t_{k}$.

The nodes of the trees are labeled. Labels may be constants, node variables (corresponding to any node value), or path variables (corresponding to any path). Constants correspond to element, attribute and text values. Nodes labeled with text-values are called text nodes and are always leaf nodes. Attribute nodes can have only one child node, a text node. Also, any two attribute nodes of a given element cannot have same label. Element nodes can have zero or more child nodes that can be elements, attributes, or text nodes. We denote element nodes with $n_{i}$, attribute nodes with $a_{i}$, and text nodes with pcdata. A labeled tree is called a ground tree if all of its nodes are labeled with constants.

Definition 3.2 (Path-expression)

Let $p=\left\{n,\left\{a_{1}, \ldots, a_{j}\right\}\right\}$ represent a single node $n$ and its child nodes corresponding to attributes $a_{1}, \ldots, a_{j}$, where $n$ is either a constant, or a variable. A path-expression is defined as: 1. $p$ is a path expression, 2. $\left\{p_{1} \rightarrow p_{2} \rightarrow \ldots \rightarrow p_{k}\right\}$ is a path-expression where $p_{i}(i=1, \ldots, k)$ are path-expressions, 3 . Let $/ /$ denote an arbitrary path-expression. Then the following are also path-expressions: $\left\{/ / \rightarrow p_{1} \rightarrow \ldots \rightarrow p_{m}\right\},\left\{p_{1} \rightarrow / / \rightarrow p_{m}\right\}$.

DTD (Bray et al., 2000) and W3C XML Schema (Thompson et al., 2001) satisfy a constraint that all child nodes of any given node must have unique names. Trees satisfying this constraint are called single-type trees (See Murata et al. (2005) for discussion on single-type tree grammars).

We consider XQuery syntax (Fernández et al., 2003) of the following form:

Definition 3.3 (XML Query)

An XML query $Q$ is of the following form:

FOR $v_{0}$ in $P_{0}$

LET $v_{1}:=P_{1}, \ldots, v_{l}:=P_{l}$

RETURN $\left\{n \rightarrow\left\{\overline{v_{k}}, \ldots, \overline{v_{j}}\right\}\right\}$

WHERE $\left(\overline{v_{i}}==\overline{v_{j}}\right.$ and $\ldots$ and $\left.\overline{v_{l}}==\overline{v_{m}}\right)$

where, $v_{i}(i=0, \ldots, l)$ are variables of query (we refer to them as query-variables in rest of this paper $), \overline{v_{i}}(i=0, \ldots, m)$ represent a path-expression $\left\{v_{i} \rightarrow p^{\prime}\right\}$ $(i=1, \ldots, l)$ and $p^{\prime}$ is a path-expression that does not contain any queryvariables, $P_{i}(i=0, \ldots, l)$ are path-expressions, and $n$ is a constant.

Given a XML query $Q$, the first step in query filtering architecture is to build query-pattern of $Q$. Let $V=\left\{v_{1}, v_{2}, \ldots, v_{l}\right\}$ be the set of query-variables defined in $Q$, and $\bar{V}=\left\{\overline{v_{1}}, \overline{v_{2}}, \ldots, \overline{v_{m}}\right\}$ be the path-expressions in the RETURN 
or WHERE clause of the query. Intuitively, the query-pattern is constructed by uniting the path-expressions in $\bar{V}$. Since path-expressions may contain queryvariables. We need a method to eliminate query variables. A formal definition of variable-substitution follows.

Definition 3.4 (Variable-Substitution)

Let $\$ v_{i}=\left\{p_{1} \rightarrow \ldots \rightarrow p_{l}\right\}$, and $\$ v_{j}=\left\{\$ v_{i} \rightarrow p_{1}^{\prime} \rightarrow \ldots p_{m}^{\prime}\right\}$ be two assignments in the FOR or LET clause of the XML query. A variable substitution replaces $\$ v_{i}$ in the second assignment with $\left\{p_{1} \rightarrow \ldots \rightarrow p_{l}\right\}$.

Example 3.1 Consider the single-type tree $T=\{\$ x \rightarrow\{a, d\}\}$, where $\$ x=$ $\{/ / \rightarrow\{r\}\}$ is a query-variable. Substituting $\$ x$, we get $T=\{/ / \rightarrow\{r \rightarrow$ $\{a, d\}\}\}$.

Definition 3.5 (Single-type Tree-Merge)

Let $P_{1}=\left\{n_{1}^{1} \rightarrow n_{2}^{1} \rightarrow \ldots n_{k}^{1} \rightarrow n_{k+1}^{1} \rightarrow \ldots n_{l}^{1}\right\}$ and $P_{2}=\left\{n_{1}^{2} \rightarrow n_{2}^{2} \rightarrow\right.$ $\left.\ldots n_{k}^{2} \rightarrow n_{k+1}^{2} \rightarrow \ldots n_{m}^{2}\right\}$ be two ground path-expressions over the same schema. We define merge of path expressions as follows:

if $n_{1}^{1}=n_{1}^{2}, n_{2}^{1}=n_{2}^{2}, \ldots, n_{k}^{1}=n_{k}^{2}$, and $n_{k+1}^{1} \neq n_{k+1}^{2}$, then $P_{1} \cup_{S} P_{2}=\left\{n_{1}^{1} \rightarrow n_{2}^{1} \ldots n_{k}^{1} \rightarrow\left\{\left\{n_{k+1}^{1} \rightarrow \ldots \rightarrow n_{l}^{1}\right\},\left\{n_{k+1}^{2} \rightarrow \ldots \rightarrow n_{m}^{2}\right\}\right\}\right.$.

We extend the notion of merging paths to merging single-type trees. Let $T_{1}=\left\{n \rightarrow\left\{t_{1}, t_{2}, \ldots, t_{k}\right\}\right\}$ and $T_{2}=\left\{n^{\prime} \rightarrow\left\{t_{1}^{\prime}, t_{2}^{\prime}, \ldots, t_{l}^{\prime}\right\}\right\}$ be two trees, then their merger $T_{1} \cup_{S} T_{2}$ is defined as follows:

1. $T \cup_{S}\{\} \stackrel{\text { def }}{=}\{\} \cup_{S} T \stackrel{\text { def }}{=} T$

2. if $n \neq n^{\prime}, T_{1} \cup_{S} T_{2}=\left\{T_{1}, T_{2}\right\}$,(trees cannot be merged).

3. if $n=n^{\prime}$, then let $T=\{\}$. For all paths $p$ originating from the root in $T_{1}$ and $T_{2}$, do $T=T \cup_{S} p . T_{1} \cup_{S} T_{2}=T$.

The query-pattern of an XML query $Q$ is a labeled-tree representing all data disclosed by $Q$. That is, all data returned to the user or accessed to evaluate the query.

Definition 3.6 (Query-Pattern)

Let $Q$ be the given XML query and $P_{1}, \ldots, P_{n}$ are path-expressions that occur in the RETURN or the WHERE clause of $Q$. If $P_{i}==P_{j}$ is a condition in the WHERE clause, we add a new leaf node labeled with a data-variable $v$ to $P_{i}$ and $P_{j}$. Substitute all query-variables in $P_{1}, \ldots, P_{n}$. Query pattern $P$ is the labeled-tree produced by merging paths $P_{1}, \ldots, P_{n}$. Algorithm 1 shows the construction of the query-pattern.

Example 3.2 Consider the following XML query $Q_{3.2}$ : FOR $\$ \mathrm{x}$ in $/ / \mathrm{r}$ LET $\$ \mathrm{y}:=\$ \mathrm{x} / \mathrm{d}, \$ \mathrm{z}:=\$ \mathrm{x} / \mathrm{a}$ RETURN < answer $>\{\$ z / c\}</$ answer $>$ WHERE \{ $\$ \mathrm{z} / \mathrm{b}==\$ \mathrm{y} \quad$. Let $T_{r}$ be the tree in the return statement of Query $Q_{3.2} . T_{r}$ specifies structure of query answer being returned to the user. To evaluate the query-answer $\$ z / b$ and $\$ y$ must be accessed. Query-pattern constructed from query $Q_{3.2}$ is shown in Fig. 3(c). 


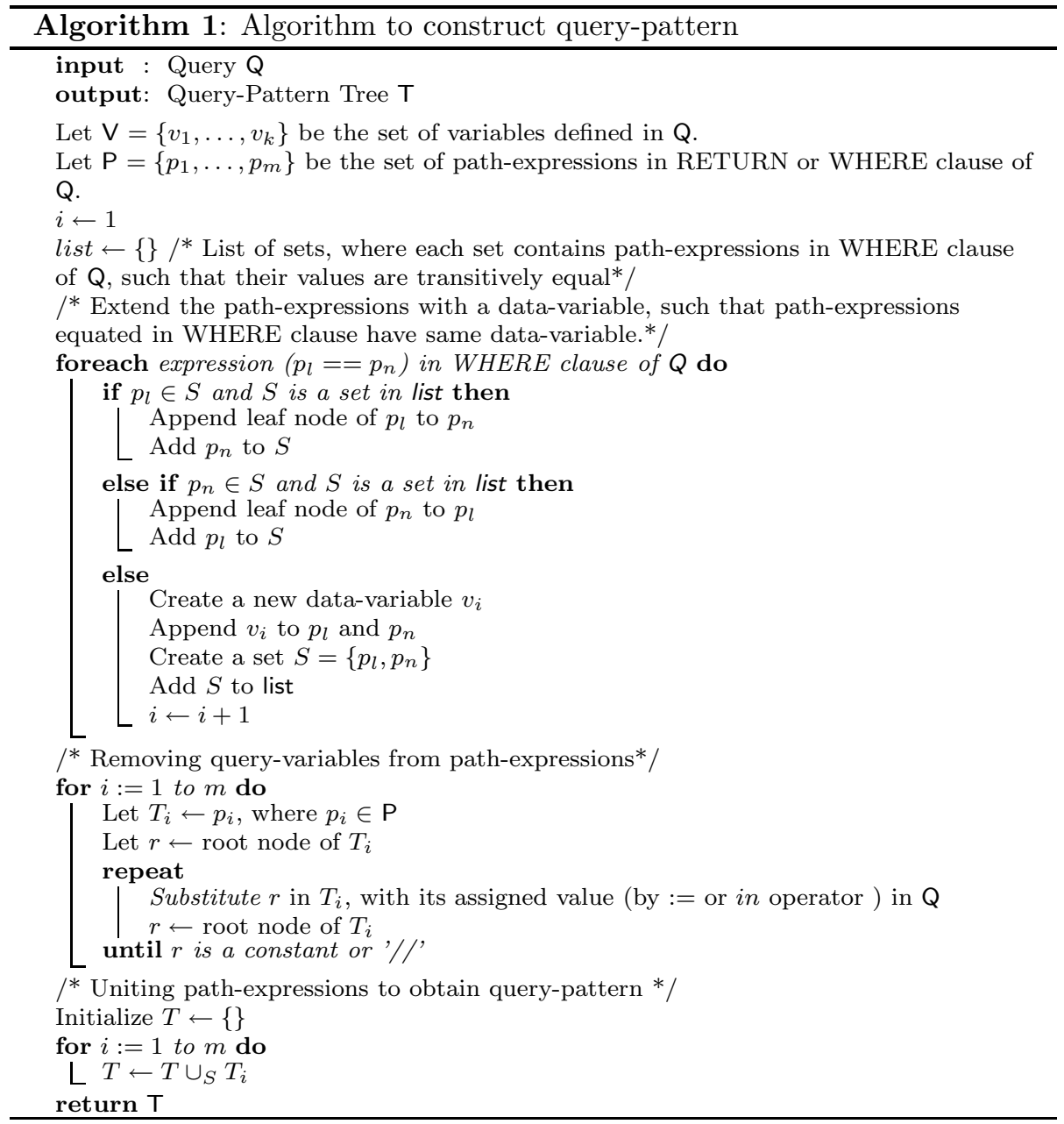

Definition 3.7 (Protection Object)

A simple security object $o$ is a node-labeled tree, where all distinct subtrees $t_{1}, t_{2}, \ldots, t_{k}$ of $o$ have the same access permission as $o$. That is, for every proper subtree $t_{i} \in o, \lambda(o)=\lambda\left(t_{i}\right)$, where $\lambda(o)$ and $\lambda\left(t_{i}\right)$ denote the security classification of $o$ and $t_{i}$ respectively. Simple security objects are equivalent to node-level security classification. An association security object $o$ is a nodelabeled tree where every proper subtree $t_{i} \in o, \lambda(o)>\lambda\left(t_{i}\right)(i=1, \ldots, n)$.

We construct Pattern Automata (PA) to represent security objects. Due to the page limitation, we only show the representation of association security objects, that is more difficult than the representation of the simple security object. 
Definition 3.8 (Pattern Automata)

Let $\mathrm{E}$ be a set of node-labels for elements, A be a set of node-labels for attributes, and let the label pcdata represent all text nodes. A Pattern Automata is defined as $\mathcal{X}=\left\{\Sigma, Q, q_{0}, Q_{f}, \delta\right\}$, where $Q=\left\{q_{0}, \ldots, q_{n}\right\}$ is a finite set of automaton states, $\Sigma=E \cup A \cup\{$ pcdata, //\} is automata alphabet, '//' is a symbol for self-or-descendant axis, $q_{0}$ is start state, $Q_{f} \subset Q,\left(q_{0} \notin Q_{f}\right)$ is set of accepting final states, and $\delta$ is set of state transition rules.

Let $\sigma \in \Sigma$ be the label of a scanned node $N$ in the given query pattern and therefore the next input symbol for the automata, and $Q_{c} \subseteq Q$ is set of states associated with child nodes of $N$. A valid transition is of the form, $\sigma\left(q_{i}, \ldots, q_{j}\right) \rightarrow q_{k}$, where $\left\{q_{i}, \ldots, q_{j}\right\} \subseteq Q_{c}$, and $q_{k}$ is state associated with $\mathrm{N}$ after scanning. For simplicity, we will often write transition rule in the form $\sigma\left(Q_{t}\right) \rightarrow q_{k}$, where $Q_{t}=\left\{q_{i}, \ldots, q_{j}\right\}$ is set of states required for transition. To distinguish data values from labels of elements and attributes, we write data values inside [ ]. If $\delta$ does not contain a valid transition rule, by default the state associated with the scanned node is $q_{0}$.

\section{Security Analysis of Query Pattern}

RXACL performs security analysis by evaluating query-pattern with the pattern automata corresponding to protection objects. An accepting state is reached if the protection object is disclosed by the input pattern. These automata can also be used for recognizing possible disclosure of security objects by query-patterns extended with document schema as discussed later in section 5 .

A pattern automaton $\mathcal{X}$ accepts a query-pattern $\mathcal{P}$ iff there is at least one accepting path of transitions that reads complete $\mathcal{P}$. For clarity, in this paper we allow use of wildcard symbol $(*)$ to represent any alphabet symbol. Let us now consider an example.

Example 4.1 The following automaton $\mathcal{X}_{4.1}=\left\{\Sigma, Q, q_{0}, Q_{f}, \delta\right\}$ is a XML Pattern Automata that accepts query patterns disclosing association $A_{3}$ (see Fig. 3(b)). An accepting run of this automaton on query $Q_{3.2}$ is shown in Fig. 3(d). It means that answers of $Q_{3.2}$ disclose $A_{3}$.

We now present Algorithm 2 and Procedure AddRules to generate pattern automata for associations. Given a query-pattern $P$ a pattern automaton $X$ is generated, such that on input $P^{\prime}, X$ accepts iff $P$ is contained in $P^{\prime}$. Algorithm 2 performs a bottom up traversal of the association security object (a labeledtree). At each step of traversal the label of current node is read. If the label is read for first time, it is added to pattern automaton's alphabet and a new state is also created. If the label denotes a self-or-descendant edge in the querypattern then a transition rule with a wildcard $(*)$ for read symbol is added to the pattern automata. Otherwise transition rule with symbol read at the current node is added.

Next we present Algorithm 4 and Procedure EvaluateQueryPattern) that runs a given pattern automata on a given query-pattern. The query pattern 


$$
\begin{aligned}
& Q=\left\{q_{0}, q_{a}, q_{b}, q_{c}\right\}, \\
& \Sigma=\{a, b, c, / /\}, \\
& q_{0}=q_{0}, \\
& Q_{f}=\left\{q_{a}\right\}, \\
& \delta=\left\{\begin{array}{c}
b() \rightarrow q_{b}, \\
c() \rightarrow q_{c}, \\
\\
\quad a\left(q_{b}, q_{c}\right) \rightarrow q_{a}, \\
\left.\quad *\left(q_{a}\right) \rightarrow q_{a}\right\}
\end{array}\right.
\end{aligned}
$$

(a)

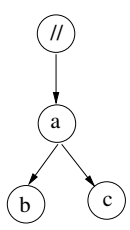

(b)

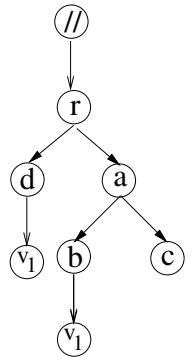

(c)

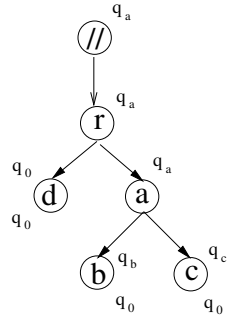

(d)

Figure 3: (a) Pattern automata example $\mathcal{X}_{4.1}$ (b) Example association $A_{3}$ (c) Query-pattern of $Q_{3.2}$ (d) States of $\mathcal{X}_{4.1}\left(q_{i}\right)$ on query-pattern of $Q_{3.2}$ as input.

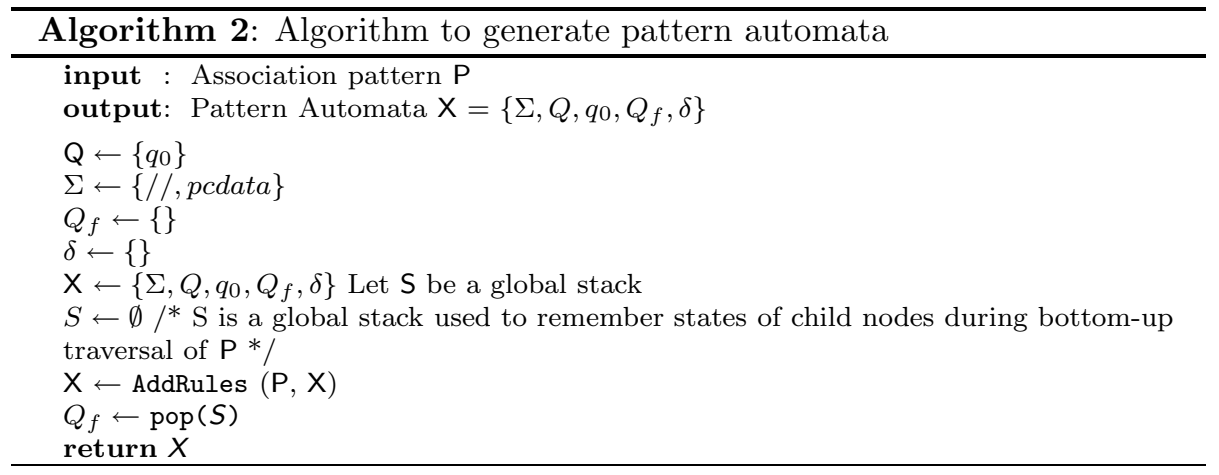

is accepted if the association object represented by the pattern automata is disclosed by the query pattern. A query-pattern may contain variables (called data-variables) at the leaves, due to the equalities in the WHERE clause. Datavariables of a query may correspond to pcdata constants of a pattern automata, thus indicating potential disclosure. The procedure EvaluateQueryPattern is data independent. Algorithm 4 analyzes the input query pattern by analyzing all possible transitions of the pattern automata in parallel. If there is at least one accepting run of the automata then the algorithm returns true, otherwise false.

Theorem 4.1 Let $Q$ be an XML query, $P$ the query-pattern generated from $Q$ (Def. 3.6), $O$ an association object and $A O$ the association-automata representing $O$. The association-automata $A O$ accepts a input query-pattern $P$ iff there exists an XML instance $I$ such that the answer to $Q$ over $I$ discloses $O$.

Proof Sketch: $(\Rightarrow)$ The pattern-automata performs bottom-up traversal of $P$, i.e., states of child nodes are evaluated before evaluating state for root node. Let $n$ be a node in $P$ scanned to detect disclosure of $O$. If $n$ is a leaf node in 

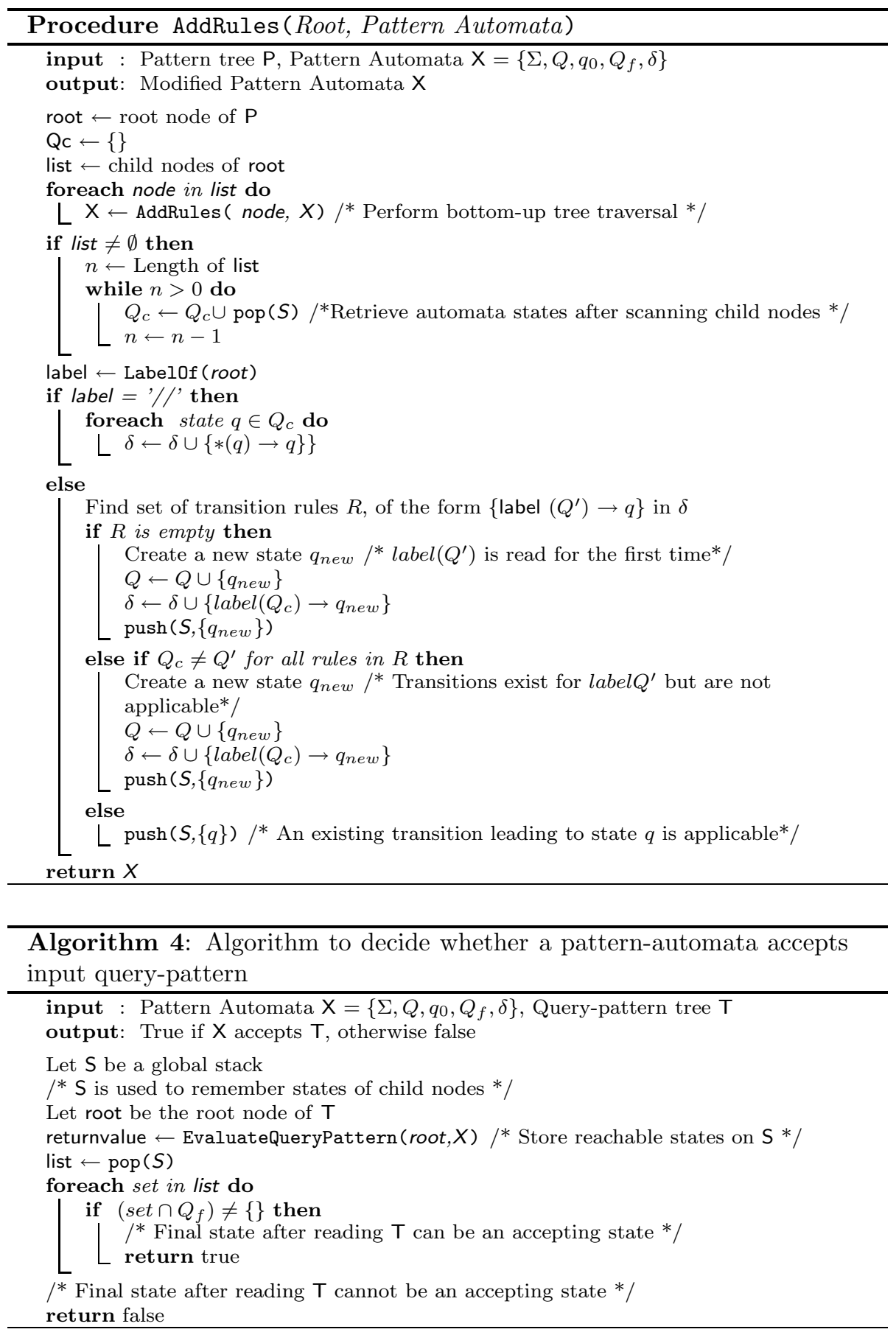


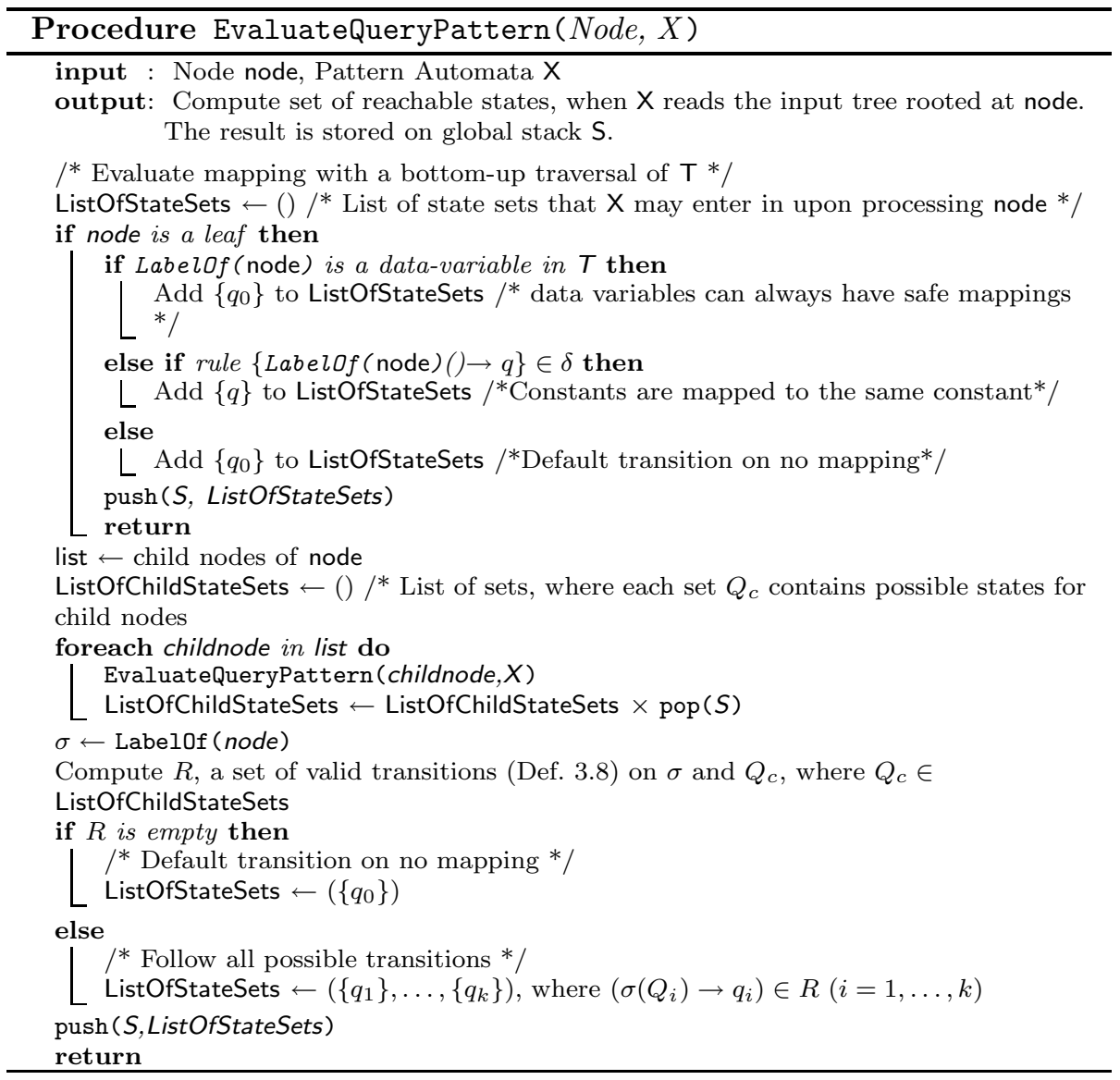

$O$, there must exist a valid transition of form $\{n() \rightarrow q\} \in \delta$, where $\delta$ is the transition function of pattern-automata $A O$ created by Algorithm 2. If $n$ is an internal node with child nodes $\left\{n_{1}, \ldots, n_{k}\right\}$, Algorithm 2 generates a transition rule of the form $\left\{n\left(q_{1}, \ldots, q_{k}\right) \rightarrow q\right\}$, where $q_{1}, \ldots, q_{k}$ are states associated with $n_{1}, \ldots, n_{k}$ respectively. Clearly there exists an accepting path of automata evaluation if the association pattern is traversed. Thus, pattern-automata finds the accepting path if it exists.

$(\Leftarrow)$ For this, we show how to construct instance $I$ such that the answer to $Q$ over $I$ must contain $O$. Let $\zeta$ be a mapping from $P$ to $O$ with following properties: $\zeta$ maps (1) a constant to the same constant, (2) variable to pcdata , and (3) a arbitrary path $p$ to //.

If there exists a $\zeta$ such that the pattern $P^{\prime}$ created from $P$ by replacing all variables of $P$ with $\zeta(v)$ and $p$ with //, and $O$ is a subtree of $P^{\prime}$ then we generate $I$ as follows: (1) replace all mapped variables $v \in P$ with $\zeta(v),(2)$ replace all non-mapped variables in $P$ with pcdata $c$, and (3) replace // with the empty path, i.e., remove //. 


\section{Security Analysis of Extended Query-Pattern}

In addition to the structural information contained in the RETURN and the WHERE clauses of the query, a query answer also contains subtrees of the original XML document, where each returned subtree originates from one of the path-expressions in the RETURN clause. To incorporate this knowledge in our model, we define the notion of extended query-pattern.

Definition 5.1 (Extended Query-Pattern)

Let $P$ denote a query-pattern and $S$ the schema (ground-tree) of the XML document that $Q$ is posed on. The extended-query-pattern (EQP) is defined as a set of trees $\left\{T_{1}, \ldots, T_{m}\right\}$, where $T_{i}(i=1, \ldots, m)$ are constructed as follows: Let $\nu$ denote a symbol mapping from the symbols of $P$ to the symbols of $S$ such that:

- for constants $\nu$ is an identity mapping.

- $\nu$ maps the data-variables to the empty node $\emptyset$.

- $\nu$ maps '//' to any ground path in $S$.

We extend $\nu$ to map paths of $P$, such that given a path $p=\left\{n_{1} \rightarrow n_{2} \rightarrow \ldots \rightarrow\right.$ $\left.n_{l}\right\}$, its mapping $\nu(p)=\left\{\nu\left(n_{1}\right) \rightarrow \nu\left(n_{2}\right) \rightarrow \ldots \rightarrow \nu\left(n_{l-1}\right) \rightarrow t_{l}\right\}$, where $t_{l}$ is a tree rooted at $\nu\left(n_{l}\right)$ such that $\nu(p) \in S$. Finally, given paths $p_{1}, \ldots, p_{k}$ of all leaf nodes in $P$ we construct $T_{i} \in E Q P$ as $T_{i}=\nu\left(p_{1}\right) \cup_{S} \nu\left(p_{2}\right) \cup_{S} \ldots \cup_{S} \nu\left(p_{k}\right) \cup_{S}$ $p_{1} \cup_{S} \ldots \cup_{S} p_{k}$ and $T_{i} \in S$ for all possible symbol mapping $\nu$.

Algorithm 6 and Procedure EvaluateExtendedQueryPattern decide whether a pattern automata accepts the extended query pattern. Unlike Procedure EvaluateQueryPattern, Procedure EvaluateExtendedQueryPattern evaluates data variables too. We now extend our formalism to be able to evaluate data variables in the query pattern.

Definition 5.2 (Self-or-ancestor state set)

Let $\mathcal{X}=\left\{\Sigma, Q, q_{0}, Q_{f}, \delta\right\}$ a XML Pattern Automata, and $T$ be an arbitrary XML pattern given as input. We say that self-or-ancestor state set $Q_{a N}=$ $\left\{q_{1}, q_{2}, \ldots, q_{k}\right\}$ is a set of states, such that $Q_{a N} \subseteq Q$, and $\mathcal{X}$ may enter a state $q_{i}$ by scanning a node labeled $N$ in $T$ or any ancestor node of $N$, iff $q_{i} \in Q_{a N}$.

Definition 5.3 (Data-characteristic state set)

Let $\mathcal{X}=\left\{\Sigma, Q, q_{0}, Q_{f}, \delta\right\}$ a XML Pattern Automata, and $T$ be an arbitrary XML pattern given as input. Data-characteristic state set $Q_{d}=\left\{q_{1}, q_{2}, \ldots, q_{k}\right\}$ is a set of states, such that $Q_{d} \subseteq Q$ and $\mathcal{X}$ can enter a state $q_{i} \in Q_{d}$ after scanning a node $N$ in $T$, iff exactly one pcdata constant occurs in subtree under node $N$.

Definition 5.4 (Variable-characteristic state set)

Let $\mathcal{X}=\left\{\Sigma, Q, q_{0}, Q_{f}, \delta\right\}$ a XML Pattern Automata, and $T$ be an arbitrary 
XML pattern with data-variables $V=\left\{v_{1}, v_{2}, \ldots, v_{k}\right\}$. For any variable $v_{i} \in V$, its variable-characteristic state set $Q_{v_{i}}=\left\{q_{1}, \ldots, q_{k}\right\}$ is a set of states, such that $Q_{v_{i}} \subseteq Q$, and $\mathcal{X}$ may enter $q_{i}$ on scanning node (in $T$ ) labeled $v_{i}$ or on scanning an ancestor node of such node.

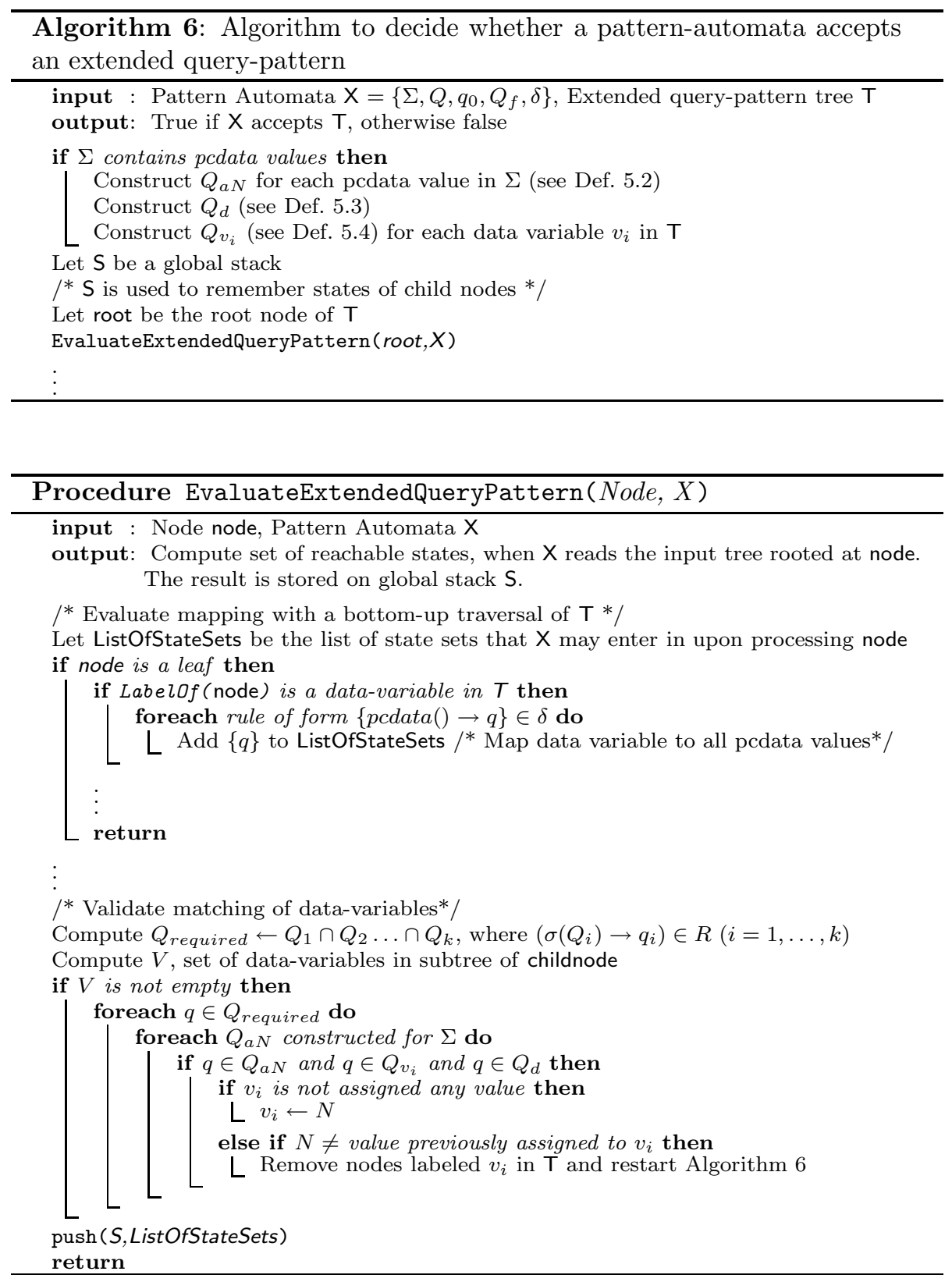


Theorem 5.1 Let $Q$ be an XML query, $S$ be the schema of XML document, $E Q P$ be the query-pattern extended with $S, O$ an association object, and $A O$ be the association-automaton representing $O$. If $A O$ does not accept the extended query-pattern $E Q P$, then the query is safe to answer for any XML document that satisfies $S$. That is for all XML instances over $S$ the query $Q$ will not disclose $O$.

Proof Sketch: Lets assume by contradiction that the query $Q$ discloses an association object $A O$ and the pattern-automata generated from $A O$ does not accept the extended query-pattern. But then, either the specifying query itself discloses $O$, i.e., the union of the paths $p_{1}, \ldots, p_{k}$ in the FOR, LET, RETURN, and WHERE clause of $Q$ disclose $O$, or the answer generated from any XML instance conforming to $S$ together with $p_{1} \cup_{S} p_{2} \cup_{S} \ldots \cup_{S} p_{k}$ disclose $O$. But this is exactly the information used to generate the extended query-pattern. Using Theorem 1 this implies that the tree-automata must accept the extended query-pattern, which is a contradiction.

\section{Conclusions}

In this paper we present a bottom-up tree automata (pattern-automata) based technique for filtering XML association before query evaluation. We give algorithms for constructing query-pattern, pattern automata, and the detection of disclosure of an association security object in a query-pattern itself. In addition we extend the query-pattern with schema information to evaluate all data that the query answer may reveal to the user. We also show that our security-analysis is complete, i.e., our method detects all possible disclosures.

We have considered only simple XQueries in this work. In our future work, we hope to extend our analysis to incorporate nested queries. At present our schema-level analysis requires the schema to be a single-type tree language (DTD or W3C XML schema). In future work we hope to extend our schemalevel security analysis to incorporate regular tree languages, such as RELAX NG. Finally, we plan to integrate query-optimization with security analysis.

\section{Acknowledgments}

This work was partially supported by National Science Foundation grant number IIS-0237782.

\section{References}

\section{References}

Bertino, E., Castano, S. and Ferrari, E. (2001): Securing XML Documents with Author-X. IEEE Internet Computing 5(3):21-31. 
Bertino, E., Castano, S., Ferrari, E. and Mesiti, M. (1999): Controlled Access and Dissemination of XML Documents. In Proc. of 2nd ACM Workshop on Web Information and Data Management, 22-27, Kansas City.

Bertino, E., Castano, S., Ferrari, E. and Mesiti, M. (2000): Specifying and enforcing access control policies for XML document sources. World Wide Web 3(3):139-151.

Bray, T., Paoli, J. and Sperberg-McQueen, C. M. (2000): Extensible Markup Language Language 1.0 specification. World Wide Web Consortium, w3c recommendation edition. http://www.w3.org/TR/2000/REC-xml20001006. Accessed 06-Oct-2000.

Clark, J. and DeRose, S. (1999): XML Path Language (XPath) Version 1.0. World Wide Web Consortium, w3c recommendation edition. http://www.w3.org/TR/xpath. Accessed 27-Jun-2001.

Damiani, E., di Vimercati, S. D. C., Paraboschi, S. and Samarati, P. (2000): XML Access Control Systems: A Component-Based Approach. In Proceedings of the IFIP WG11.3 Working Conference on Database Security, 39-50, The Netherlands.

Dridi, F. and Neumann, G. (1998): Towards Access Control for Logical Document Structure. In Proc. of the Ninth International Workshop of Database and Expert Systems Applications, 322-327, Vienna, Austria.

Fernández, M., Malhotra, A., Marsh, J., Nagy, M. and Walsh, N. (2003): XQuery 1.0 and XPath 2.0 Data Model. World Wide Web Consortium, working draft edition. http://www.w3.org/TR/query-datamodel/. Accessed 12-Dec-2003.

Gabillon, A. and Bruno, E. (2002): Regulating access to XML documents. In Das'01: Proceedings of the fifteenth annual working conference on Database and application security, 299-314, Norwell, MA, USA: Kluwer Academic Publishers.

Gowadia, V. and FARKas, C. (2003): RDF metadata for XML Access Control. In Proceedings of the 2003 ACM workshop on XML security, 39-48, ACM Press.

Kudo, M. and HADA, S. (2000): XML document security based on provisional authorization. In CCS '00: Proceedings of the 7th ACM conference on Computer and communications security, 87-96, New York, NY, USA: ACM Press.

Luo, B., Lee, D., Lee, W.-C. and Liu, P. (2004): QFilter: fine-grained run-time XML access control via NFA-based query rewriting. In CIKM '04: Proceedings of the Thirteenth ACM conference on Information and knowledge management, 543-552, New York, NY, USA: ACM Press. 
Murata, M., Lee, D., Mani, M. and Kawaguchi, K. (2005): Taxonomy of XML Schema Languages using Formal Language Theory. ACM Trans. on Internet Technology .

Murata, M., Tozawa, A., Kudo, M. and Hada, S. (2003): XML Access Control using Static Analysis. In CCS '03: Proceedings of the 10th ACM conference on Computer and communications security, 73-84, ACM Press.

Thompson, H. S., Beech, D., Maloney, M. and Mendelsohn, N. (2001): XML Schema Part 1: Structures. Technical report, W3C Consortium. http://www.w3.org/TR/2001/REC-xmlschema-1-20010502/. Accessed 12-May-2005. 